GENE 06801

Short Communications

\title{
A small plasmid for recombination-based screening
}

(Escherichia coli; R6K; recombinant DNA; supF; suppressor)

\author{
Andrzej J. Hanzlik, Malgorzata M. Osemlak-Hanzlik and David M. Kurnit
}

Departments of Pediatrics and Human Genetics, Howard Hughes Medical Institute, University of Michigan Medical Center, Ann Arbor, MI 48109-0650, USA

Received by J.L. Slightom: 9 June 1992; Accepted: 12 July 1992; Received at publishers: 20 August 1992

\section{SUMMARY}

We reported recently the construction of the 4.4-kb R6K-derived pMAD1 plasmid carrying sup $F$ [Stewart et al., Gene 106 (1991) 97-101] that does not share nt sequences with ColE1 and therefore permits recombination-based screening of $\lambda$ libraries that contain ColE1 sequences. Here we describe the construction of the $2.5-\mathrm{kb}$ R6K-derived plasmid, pMAD3, that lacks the $\pi$-encoding pir gene required for R6K replication. To supply $\pi$ [Inuzuka and Helinski, Proc. Natl. Acad. Sci. USA 75 (1978) 5381-5385] in trans, we employed pPR1422pir 116, referred to henceforth as pPR1 [McEachern et al., Proc. Natl. Acad. Sci. USA 86 (1989) 7942-7946; Dellis and Filutowicz, J. Bacteriol. 173 (1991) 1279-1286]. Plasmid pMAD3 is small enough to be amplified readily by PCR [Saiki et al., Science 230 (1985) 1350-1354]. This permits the insertion of larger fragments and the retrieval of larger $\lambda$ inserts, as well as the use of a simplified PCR-based cloning protocol which utilizes annealing rather than ligation to create recombinants in pMAD3 [Nisson et al., PCR Methods and Applications 1 (1991) 120-123].

\section{INTRODUCTION}

A feature of the recombination-based assay (Seed, 1983; Kurnit and Seed, 1990) is that the $\operatorname{supF}$ plasmid is integrated into bacteriophage $\lambda$ carrying an insert that shares nt sequences with the insert in the plasmid. The size constraint of phage packaging mandates that decreasing the size of the integrated plasmid yields more space for the

Correspondence to: Dr. D.M. Kurnit, Howard Hughes Medical Institute, University of Michigan Medical Center, $1150 \mathrm{~W}$. Medical Center Dr., Ann Arbor, MI 48109-0650, USA. Tel. (313)747-4747;

Fax (313) $936-9353$.

Abbreviations: bp, base pair(s); E., Escherichia; kb, kilobase(s) or $1000 \mathrm{bp}$; $\mathrm{Km}$, kanamycin; ${ }^{\mathrm{M}}$, modification; MCS, multiple cloning site (polylinker); $\mathrm{nt}$, nucleotide(s); ori, origin of DNA replication; PCR, polymerase chain reaction; pfu, plaque-forming unit(s); $\pi$, protein required for plasmid $\mathrm{R} 6 \mathrm{~K}$ (and pMAD) replication; pir, gene encoding $\pi^{;}{ }^{\mathbf{R}}$, resistance/resistant; ss, single strand(ed); $s u p F$, gene encoding mutant tyrosine tRNA that translates an amber stop codon; Tc, tetracycline; [ ], denotes plasmid-carrier state. inserts. Here we describe the construction of a small supF plasmid that is achieved by placing an essential plasmid gene on an ancillary helper plasmid.

\section{EXPFRIMFNTAI AND DISCUSSION}

\section{(a) Construction of pMAD3}

To screen by recombination a variety of $\lambda$ libraries that contain ColE1 sequences, we elaborated the nonhomologous plasmid, pMAD1. This 4.4-kb plasmid, unrelated to either $\lambda$ or ColE1, carries an $\mathrm{R} 6 \mathrm{~K}$ ori, a $\mathrm{Km}^{\mathrm{R}}$ marker, a polylinker, and the R6K pir gene (Stewart et al., 1991). An increased copy number of this plasmid was obtained by introducing the single bp pir-41 mutation into the $\pi$ gene (Inuzuka and Wada, 1985). We have used pMADl to study the transcription pattern of multiple genomic sequences (A.J.H., M.M.O.-H., M.A. Hauser, M. Van Keuren and D.M.K., unpublished).

To construct a smaller derivative of PMADl, we utilized a helper plasmid, pPR1, which does not share nt sequences 


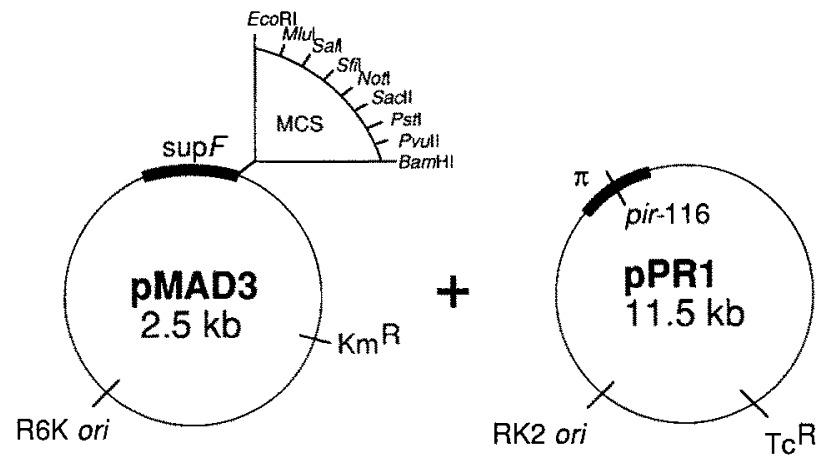

Fig. 1. Plasmids pMAD3 and pPR1. The components of these plasmids are: pMAD3 carries the $\mathrm{R} 6 \mathrm{~K}$ ori, a $\mathrm{Km}^{\mathrm{R}}$ gene, a sup $F$ gene, and a useful MCS; pPR1 is an RK2 derivative that carries a $\pi$-encoding pir gene with a pir-116 mutation which confers a high copy number onto pMAD3.

with either ColE1-based or R6K-based plasmids. This helper plasmid supplies the $\pi$ protein of $\mathrm{R} 6 \mathrm{~K}$ in trans, which enabled us to eliminate the pir gene (and approx. $2 \mathrm{~kb})$ from pMAD1. The small size $(2.5 \mathrm{~kb})$ of the resulting pMAD3 vector enabled us both to amplify pMAD3 by PCR readily and to put in larger inserts and/or retrieve larger clones by recombination than with pMAD1 (Fig. 1).

To construct pMAD3, we amplified pMAD1 with the primers pMAD-380 and pMAD-1990 (Table I), yielding a 2.5-kb PCR product (the numbers 380 and 1990 correspond to the nt in the partial Ecpr6k sequence of R6K deposited in the GenBank, accession number V00320). This PCR product included the $\mathrm{R} 6 \mathrm{~K} \gamma$ ori, supF, $\mathrm{Km}^{\mathrm{R}}$, and the polylinker of pMAD1 (Stewart et al., 1991) but lacked the $\beta$ ori and the essential $\pi$ protein of R6K which was supplied in trans by pPR 1 (McFachern et al., 1989; Dellis and Filutowicz, 1991). Plasmid pPR1 is an 11.5-kb RK2derived $\mathrm{Tc}^{\mathrm{R}}$ plasmid which expresses a $\pi$ protein with increased activity due to the single bp pir-116 mutation (Filutowicz et al., 1986, 1987), analogous to the pir-41 mutation which was present in pMAD1 (Stewart et al., 1991). We moved the $T c^{R}$ pPR 1 plasmid carrying the pir gene into the nonrestricting $h s d M^{+} h d s R^{-}$strain, DM1061, a tond $A^{-}$derivative of MC1061 (Casadaban and Cohen, 1980). (Be-

\section{TABLE I}

Sequences of primers

LsupF: 5'-ATTGTTTCGGACTTTTGAA-3'

pMADins: 5 '-CAGATCCGGAATTGGATC -3

igt 11 U-1: 5 -(CUA) ${ }_{4}$ GCCCGTCAGTATCGGCGG-3'

$\lambda$ gt 11 U-2: $5^{\prime}$-(CUA) ${ }_{4}$ AGCCCGGCGCTCAGCTGG-3'

pMAD-380: 5 ' -(CUA) $)_{4}$ AAGCTAGATCTGAAGATC -3

PMAD-1990: $5^{\prime}$-(UAG) ${ }_{4}$ GCGGTAGATCTATGGATGTTG-3'

pMAD U-4: $5^{\prime}$-(UAG) $)_{4}$ CTGGATCCAATTCCGGATCTG-3

PMAD U-5: 5 -(UAG) ${ }_{4}$ TTCTTTCTCAACGTAACACTTTAC-3' cause DM1061 is a tonA ${ }^{-}$derivative of MC1061, it lacks the receptor for and therefore does not plate $\mathrm{Tl}$ phages.) DM1061 has the additional advantage of being $\mathrm{mcr}^{-}$ $m c r B^{-}$(Raleigh and Wilson, 1986; Raleigh et al., 1988), so it is an excellent host lacking multiple $E$. coli restriction activities.

To obtain pMAD3 from PMAD1 by PCR, conditions for amplification of a pMAD1 template were $50 \mathrm{~s}$ at $94^{\circ} \mathrm{C}$ for denaturation, $50 \mathrm{~s}$ at $58^{\circ} \mathrm{C}$ for annealing, and $3 \mathrm{~min}$ at $72^{\circ} \mathrm{C}$ for extension for 35 cycles followed by a final extension time of $7 \mathrm{~min}$. This yielded an expected product of $2.5 \mathrm{~kb}$ visualized on a $0.8 \%$ agarose (Seakem) gel. The product was ethanol precipitated, dried, resuspended in UDG buffer ( $25 \mathrm{mM}$ Tris. $\mathrm{HCl} \mathrm{pH} 7.8 / 10 \mathrm{mM} \mathrm{MgCl}_{2} /$ $4 \mathrm{mM} \beta$-mercaptoethanol $/ 0.4 \mathrm{mM} \mathrm{ATP}$ ) at a concentration of $500 \mathrm{ng}$ in $20 \mu \mathrm{l}$, and treated with 1 unit of uracil DNA glycosylase (UDG; BRL) for $2 \mathrm{~h}$ at room temperature. The UDG was then heat inactivated at $65^{\circ} \mathrm{C}$ for 20 min (Nisson et al., 1991). The resulting complementary ss ends were annealed at room temperature for $1 \mathrm{~h}$ to form a $\mathrm{Km}^{\mathrm{R}}$ open circle. Of this solution, $2 \mu \mathrm{l}$ was used to transform the $\mathrm{Tc}^{\mathrm{R}}$ strain, DM1061[pPR1]. The resulting $\mathrm{Km}^{\mathrm{R}}$ $\mathrm{Tc}^{\mathrm{R}}$ colonies were screened by PCR using primers pMAD U-4 and pMAD U-5 (Table I) to confirm the isolation of a DM1061[pPR1] colony carrying the 2.5 -kb pMAD3.

\section{(b) Use of the vector pMAD3}

CsCl-purified DNA from DM1061[pMAD3][pPR1] was digested with EcoRI. Linear pMAD3 DNA was PCR amplified using the (UAG) $)_{4}$-containing primers pMAD U-4 and pMAD U-5 (Table I) to yield a 2.5 -kb product. This 2.5-kb linear molecule was isolated from a low-meltingpoint agarose gel by phenol extraction followed by ethanol precipitation with carrier $0.025 \%$ linear acrylamide $(\mathrm{w} / \mathrm{v})$, resuspended in UDG buffer (Nisson et al., 1991), and treated with UDG as described above. The resulting plasmid with ss tails can then be stored at $4^{\circ} \mathrm{C}$.

\section{(c) Preparation of insert}

The sequence to be cloned by the above strategy, PG1, was an insert in $\lambda$ gt 11. This insert was PCR amplified using primers $\lambda$ gt $11 \mathrm{U}-1$ and $\lambda$ gt $11 \mathrm{U}-2$ (Table I) that each contain a $5^{\prime}-(\mathrm{CUA})_{4}-3^{\prime}$ sequence (Nisson et al., 1991). After PCR amplification, the 700-bp insert was isolated from a low-melting-point agarose gel as described above and treated with UDG, resulting in the generation of ss overhangs complementary to those of the vector (Nisson et al., 1991).

\section{(d) Annealing of vector and insert}

Equal volumes of the UDG-treated $\mathrm{Km}^{\mathrm{K}}$ pMAD3 vector (approx. $10 \mathrm{ng} / \mu \mathrm{l}$ ) and the UDG-treated insert (also approx. $10 \mathrm{ng} / \mu \mathrm{l})$ were mixed and allowed to anneal at 
room temperature for $1 \mathrm{~h}$. The mixture was then used to transform competent $\mathrm{Tc}^{\mathrm{R}}$ DM1061[pPR1] to $\mathrm{Km}^{\mathrm{R}} \mathrm{Tc}^{\mathrm{R}}$. Inserts in the resulting colonies were analyzed by amplification with primers located in supF and the polylinker (primers LsupF and pMADins; Table I) that are 250-bp apart. Thus, clones with no insert yielded a 250 -bp fragment, whereas clones containing an insert yielded a larger fragment.

By virtue of amplification using LsupF and pMADins primers, we demonstrated that the expected 700 -hp insert from phage PG1 was inserted into pMAD3. To compare the efficiency of pMAD3 with pMAD1, this PCR product was also digested with $E c o$ RI and placed into the EcoRI site of pMAD1. This enabled us to compare the recombination rates mediated by the PG1 insert in PMAD1 and pMAD3.

To extend this comparison between pMAD1 and pMAD3, we transformed clone 4D12 from YAC A125B12 on chromosome 21 in pMAD1 [which we demonstrated previously by recombination (Seed, 1983; Kurnit and Seed, 1990; and unpublished data) to represent an expressed sequence in pMAD1 (Stewart et al., 1991)] into pMAD3. Using primers pMAD-380 and pMAD-1990, we followed the deletion strategy described above to construct $4 \mathrm{D} 12$ inserted into the equivalent of pMAD3.

\section{(e) Selection using the recombination-based assay}

Recombination (Seed, 1983; Kurnit and Seed, 1990) was performed between the $\lambda$ phage carrying PG 1 and the same PG1 insert in both pMAD1 and pMAD3. This was done by lysing overnight $0.2-\mathrm{ml}$ cultures of pMAD 1 and pMAD3 carrying the PG1 insert with $10^{6} \mathrm{PGl}$ phages, followed by plate elution with $3 \mathrm{ml}$ of $\mathrm{SM}$ buffer $(0.1 \mathrm{M} \mathrm{NaCl} / 8 \mathrm{mM}$ $\mathrm{MgSO}_{4} / 0.5 \mathrm{mM}$ Tris $\cdot \mathrm{HCl} \mathrm{pH} 7.5 / 0.01 \%$ gelatin). Homologous phages, which acquired $s u p F$ via recombination, were detected as plaques on the selective strain DM21 (Kurnit and Sccd, 1990). In each case, the rate of recombination indicated that $10^{-2}$ phages had recombined with the insert in the plasmid, as expected for homologous sequences (Kurnit and Seed, 1990).

Furthermore, we showed by recombination using both pMAD1 and pMAD3 that insert 4D12 was expressed in cDNA libraries constructed from the RNA of human 20week-old abortus fetal spinal cord and brain. The 4D12 insert in either pMAD1 or pMAD3 was allowed to recombine with the above cDNA libraries. Approx. $10^{6} \mathrm{cDNA}$ library phages were plated on cultures of 4D 12 in pMAD1 and pMAD3. Following confluent lysis, eluates from these plates corresponding to $5 \times 10^{8}$ pfu on a nonselective strain were plated on selective strain DM21, yielding approximately four plaques per plate with the fetal spinal cord library and two plaques per plate with the fetal brain library for 4D12 cloned both in pMAD1 and pMAD3. Thus, the rates at which recombination occurred were similar for 4D12 cloned both in pMAD1 and pMAD3.

Plasmid pMAD3 is currently used in our laboratory to study the transcription profile of various genomic sequences (A.J.H., M.M.O.-H., M.A. Hauser, M. Van Keuren and D.M.K., unpublished). In summary, pMAD3 has all the features of its predecessor pMAD1, and in addition, its smaller size allows the cloning and screening of larger DNA inserts and application of PCR-based amplification to facilitate cloning. Its lack of homology to sequences in cDNA libraries makes pMAD3 an excellent choice for recombination-based screening.

\section{ACKNOWLEDGEMENTS}

D.M.K. is an Investigator, and A.J.H. and M.M.O.-H. are Associates of the Howard Hughes Medical Institute. Dr. M. Filutowicz supplied pPR1 (pPR1422pir116) and advice.

\section{REFERENCES}

Casadaban, M.J. and Cohen, S.N.: Analysis of gene control signals by DNA fusion and cloning in Escherichia coti. J. Mol. Biol. 138 (1980) 179-207.

Dellis, S. and Filutowicz, M.: Integration host factor of Escherichia coli reverses the inhibition of R6K plasmid replication by $\pi$ initiator protein. J. Bacteriol. 173 (1991) 1279-1286.

Filutowicz, M., McEachern, M.J. and Helinski, D.R.: Proc. Natl. Acad. Sci. USA 83 (1986) 9645-9649.

Filutowicz, M., McEachern, M.J., Mukhopadhyay, P., Greener, A., Yang, S. and Helinski, D.R: DNA and protein interactions in the regulation of plasmid replication. J. Cell Sci. Suppl. 7 (1987) 15-31.

Inuzuka, M. and Helinski, D.R.: Requirement of a plasmid-encoded protein for replication in vitro of plasmid R6K. Proc. Natl. Acad. Sci. USA 75 (1978) 5381-5385.

Inuzuka, $M$. and Wada, $Y:$ A single amino acid mutation in the initiation protein is responsible for the DNA overproduction phenotype of copy number mutants of plasmid R6K. EMBO J. 4 (1985) 2301-2307.

Kolter, R. and Helinski, D.R.: Construction of plasmid R6K derivatives in vitro: Characterization of the $\mathrm{R} 6 \mathrm{~K}$ replication region. Plasmid 1 (1978) $571-580$.

Kurnit, D.M. and Seed, B.: Improved genetic selection for screening bacteriophage libraries by homologous recombination in vivo. Proc. Natl. Acad. Sci. USA 87 (1990) 3166-3169.

McEachern, M.J., Bott, M.A., Tooker, P.A. and Helinski, D.R.: Negalive control of plasmid R6K replication: possible role of intermolecular coupling of replication origins. Proc. Natl. Acad. Sci. USA 86 (1989) 7942-7946.

Nisson, P.E., Rashtchian, A. and Watkins, P.C.: Rapid and efficient cloning of Alu-PCR products using uracil DNA glycosylase. PCR Methods \& Applications 1 (1991) 120-123.

Raleigh, E.A. and Wilson, G.: Escherichia coli K-12 restricts DNA containing 5-methylcytosine. Proc. Natl. Acad. Sci. USA 83 (1986) 9070-9074.

Raleigh, E.A., Murray, N.E., Revel, H., Blumenthal, R.M., Westaway, D., Reith, A.D., Rigby, P.W.J., Elhai, J. and Hanahan, D.: McrA and 
McrB restriction phenotypes of some $E$.coli strains and implications for gene cloning. Nucleic Acids Res. 16 (1988) 1563-1575.

Saiki, R.K., Scharf, S., Faloona, F., Mullis, K.B., Horn, G., Erlich, H and Arnheim, N.: Enzymatic amplification of $\beta$-globin genomic sequences and restriction site analysis for diagnosis of sickle cell anemia. Science 230 (1985) 1350-1354.
Seed, B.: Purification of genomic sequences from bacteriophage libraries by recombination and selection in vivo. Nucleic Acids Res. 11 (1983) $2427-2445$.

Stewart, G.D., Hauser, M.A., Kang, H., McCann, D.P., Osemlak, M.M., Kurnit, D.M. and Hanzlik, A.J.: Plasmids for recombination-based screening. Gene 106 (1991) 97-101. 